\title{
Opiate-Induced Molecular and Cellular Plasticity of Ventral Tegmental Area and Locus Coeruleus Catecholamine Neurons
}

\author{
Michelle S. Mazei-Robison and Eric J. Nestler \\ Fishberg Department of Neuroscience and Friedman Brain Institute, Mount Sinai School of Medicine, \\ New York, New York 10029 \\ Correspondence: eric.nestler@mssm.edu
}

\begin{abstract}
The study of neuronal adaptations induced by opiate drugs is particularly relevant today given their widespread prescription and nonprescription use. Although much is known about the acute actions of such drugs on the nervous system, a great deal of work remains to fully understand their chronic effects. Here, we focus on longer-lasting adaptations that occur in two catecholaminergic brain regions that mediate distinct behavioral actions of opiates: ventral tegmental area (VTA) dopaminergic neurons, important for drug reward, and locus coeruleus (LC) noradrenergic neurons, important for physical dependence and withdrawal. We focus on changes in cellular, synaptic, and structural plasticity in these brain regions that contribute to opiate dependence and addiction. Understanding the molecular determinants of this opiate-induced plasticity will be critical for the development of better treatments for opiate addiction and perhaps safer opiate drugs for medicinal use.
\end{abstract}

B ecause of their potent analgesic properties, opiate drugs have been used for centuries. Opiates include compounds derived from the opium poppy such as morphine and codeine, as well as many synthetic derivatives such as heroin, oxycodone, and hydrocodone. For the purposes of this review, we focus on the actions of morphine and heroin, as these have been the most studied in model systems. Despite effectiveness in treating acute pain, there are serious complications with long-term opiate use, including tolerance, physical dependence, and addiction (Ballantyne and LaForge 2007). Abuse of prescription drugs, and specifically pain-relieving opiates, has increased greatly in recent years

in both the adult and adolescent U.S. populations (Compton and Volkow 2006; Manchikanti et al. 2010). The medical use of opiates has also risen steadily as treatment for chronic pain disorders has become more aggressive (Kuehn 2007). Although the ethics of chronic pain treatment and the potential over or under use of opiate drugs can be debated (Fields 2011), there is no question that chronic opiate use causes neuroadaptations that lead to undesirable effects.

Physical dependence and addiction to opiates were once considered closely linked; however, these processes are now believed to be mediated by distinct mechanisms and circuits within the brain (Koob and Le Moal 2001). Physical

Editors: R. Christopher Pierce and Paul J. Kenny

Additional Perspectives on Addiction available at www.perspectivesinmedicine.org

Copyright (C) 2012 Cold Spring Harbor Laboratory Press; all rights reserved; doi: 10.1101/cshperspect.a012070

Cite this article as Cold Spring Harb Perspect Med 2012;2:a012070 
M.S. Mazei-Robison and E.J. Nestler

dependence is manifested as negative physical symptoms (e.g., sweating, abdominal pain, diarrhea) when the drug is withdrawn. Addiction, or "substance dependence" as defined by the Diagnostic and Statistical Manual of Mental Disorders, has a profound long-term impact on health and productivity and is characterized by the compulsion to seek and take drug despite negative consequences. Part, but not all, of this addition phenotype likely reflects "psychological dependence," that is, negative emotional symptoms that occur during drug withdrawal.

In this review, we discuss what is known about the neuroadaptations, or opiate-induced plasticity, that occur in two brain regions rich in catecholamine neurons, that play critical roles in opiate addiction and physical dependence, respectively: dopaminergic neurons within the midbrain ventral tegmental area (VTA) and noradrenergic neurons within the pontine locus coeruleus (LC). This discussion focuses on three types of opiate-induced plasticity in these regions: synaptic plasticity-persistent changes in glutamatergic and GABAergic synaptic transmission (Dacher and Nugent 2011b; Luscher and Malenka 2011); cellular plasticity-homeostatic changes in intracellular signaling cascades (Williams et al. 2001; Nestler 1992, 2004); and structural plasticity-long-lasting changes in neuronal morphology (Russo et al. 2010). Identifying the molecular determinants of these three types of plasticity in the brain's catecholaminergic neurons serves as a model of the plasticity induced in other important neural substrates of addiction and will be key to developing better therapies for opiate addiction and possibly safer opiate drugs for analgesia.

\section{VENTRAL TEGMENTAL AREA}

\section{Background}

The VTA has been widely studied in drug abuse given its fundamental role in reward. Dopamine (DA) neurons in VTA project to multiple brain regions including the nucleus accumbens (NAc), where increased DA release has been noted in response to every class of abused drug (DiChiara and Imperato 1988). However, while DA neu- rons are a prominent portion $(\sim 60 \%-65 \%)$ of this midbrain nucleus, there is considerable cellular diversity, with a significant portion of GABA neurons $(30 \%-35 \%)$ as well as descriptions of glutamatergic neurons $(2 \%-3 \%)$ (Swanson 1982; Nair-Roberts et al. 2008; Sesack and Grace 2010). The DA and GABA neurons within the ventral midbrain, in general, project topographically (medial to lateral) with the main output structures consisting of NAc, prefrontal cortex (PFC), and amygdala (AMY) (extensively reviewed in Sesack and Grace 2010) (Fig. 1). The primary afferents to VTA include excitatory inputs from PFC, pedunculopontine and laterodorsal tegmentum (PPTg and LDT), as well as many other recently defined structures (Geisler et al. 2007). The inhibitory input to the VTA is less well defined, but inputs from NAc, ventral pallidum, and mesopontine rostromedial tegmental nucleus (RMTg) have been reported (Sesack and Grace 2010). Research to date has focused disproportionately on DA neurons in VTA, and specifically those that project to NAc, because of the critical role of this projection in reward (Nestler 2004).

\section{Acute Opiate-Induced Changes in Neuronal Activity}

Given the ability of acute morphine into the VTA to elicit increased DA release in the NAc (Leone et al. 1991), a substantial amount of work has examined the acute effects of opiates in the VTA. Acute morphine increases the firing rate of DA neurons in VTA (Gysling and Wang 1983). This effect is mediated at least in part by the binding of morphine to the $\mathrm{G}_{\mathrm{i} / \mathrm{o}}$-coupled $\mu$-opioid receptor (MOR) on local GABA neurons, thereby decreasing their activity and subsequent GABA release on DA neurons and resulting in disinhibition of DA neurons (Johnson and North 1992). However, interpretation of much of the early electrophysiology work is complicated by evidence highlighting the near indistinguishable nature of VTA DA and GABA neurons (by size, morphology, and electrophysiological properties) (Margolis et al. 2006), clarifying the need to identify VTA neurons studied more definitively (e.g., by immunohistochemistry, use of 


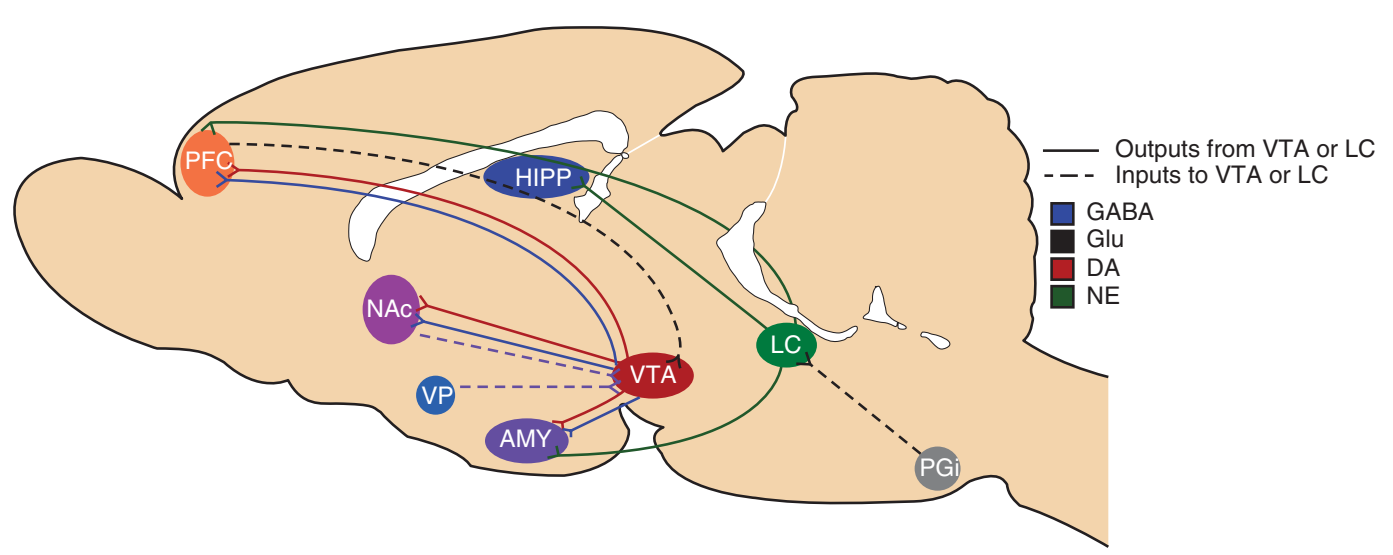

Figure 1. Cartoon of a sagittal section of rodent brain illustrating the VTA and LC and their major afferent and efferent projections. DAergic (red) and GABAergic (blue) neurons in VTA project to limbic and cortical structures and receive glutamatergic (black-dash, PFC) and GABAergic input (blue-dash, NAc, VP). Noradrenergic neurons (green) in LC innervate multiple regions including HIPP and PFC and receive glutamatergic input from PGi. Abbreviations: AMY, amygdala; HIPP, hippocampus; LC, locus coeruleus; NAc, nucleus accumbens; PFC, prefrontal cortex; PGi, nucleus paragigantocellularis; VP, ventral pallidum; VTA, ventral tegmental area.

GFP reporter mice, etc.), a point that will be discussed in detail later in this review. Here, we mainly focus on opiates that act as agonists at the MOR in VTA, such as morphine, as these drugs produce the rewarding effects most often studied in the drug abuse field. However, it is known that $\kappa$-opioid receptors (KOR) are also expressed on VTA DA neurons, and that activation of these receptors can directly inhibit the firing rate of DA neurons (Margolis et al. 2003), likely contributing to the aversive effects of kappa agonists. The ability of opiates to produce both VTA DA neuron activation and inhibition, and rewarding and aversive effects, is intriguing, and this "yin-yang" modulation and the role of endogenous opioid peptides in reward deserves to be a focus of future study.

\section{Acute Opiate-Induced Synaptic Plasticity}

In addition to changes in neuronal activity, there are many reports of synaptic plasticity induced by acute opiates. As with cocaine and other abused drugs, a single injection of morphine was found to increase the ratio of $\alpha$-amino-3hydroxy-5-methyl-4-isoxazolepropionic acid (AMPA) to $N$-methyl-D-asparticacid (NMDA) excitatory postsynaptic currents (EPSCs) 24 hours after administration, consistent with long-term potentiation (LTP) of glutamatergic synapses onto DA neurons (Saal et al. 2003). Recently, it has also been reported that acute morphine induces AMPAR receptor (AMPAR) redistribution in VTA in a manner similar to cocaine, specifically an insertion of GluA2lacking AMPARs (Brown et al. 2010). Brown et al. observed an increased rectification index and increased cytoplasmic GluA2 AMPAR in response to acute morphine, an effect that is recapitulated by direct stimulation of DA neurons in VTA using selective channelrhodposin 2 expression (Brown et al. 2010), directly implicating DA activity/signaling within VTA to glutamatergic regulation. These data are consistent with earlier work that GluA1, but not GluA2, overexpression in VTA sensitizes animals to morphine's locomotor-activating and rewarding behaviors (Carlezon et al. 1997).

Acute opiates also influence plasticity at GABAergic synapses in VTA. High-frequency stimulation has been found to elicit LTP at GABA terminals $\left(\mathrm{LTP}_{\mathrm{GABA}}\right)$ on VTA DA neurons, an effect that is dependent on activation of postsynaptic NMDA receptors (NMDAR) and release of nitric oxide (NO) as a retrograde messenger from DA neurons (Nugent et al. 2007). 
M.S. Mazei-Robison and E.J. Nestler

NO then increases guanylyl cyclase (GC) activity in the GABA neuron, leading to increased GABA release and $\mathrm{LTP}_{\mathrm{GABA}}$. A single dose of morphine inhibits LTP $_{\mathrm{GABA}}$ by interrupting the NO-GC-protein kinase G (PKG) signal cascade, causing a loss of normal inhibitory control (observed 2 and 24 hours following injection, but not 5 days) (Nugent et al. 2007, 2009; Niehaus et al. 2010). Thus, disruption of $\mathrm{LTP}_{\mathrm{GABA}}$ provides another mechanism for the ability of acute opiates to increase VTA DA neuronal activity.

More recently, another form of VTA GABAergic plasticity has been described: longterm depression of GABAergic synapses onto DA neurons $\left(\right.$ LTD $_{\mathrm{GABA}}$ ) (Dacher and Nugent 2011a). Using low-frequency stimulation (LFS), a stable LTD $_{\mathrm{GABA}}$ in DA cells was induced that, in contrast to $\mathrm{LTP}_{\mathrm{GABA}}$, was expressed postsynaptically and did not depend on NMDAR. This effect was also not dependent on endocannabinoid signaling, but was blocked by the dopamine D2 receptor (D2R) antagonist sulpiride. Interestingly, a single morphine injection was sufficient to prevent LFS-induced LTD $_{\text {GABA }} 24$ hours after administration, suggesting that morphine can bidirectionally regulate GABA plasticity in VTA (Dacher and Nugent 2011a).

\section{Chronic Opiate-Induced Synaptic Plasticity}

Although the synaptic changes that occur with acute opiates have been relatively well characterized, the chronic changes have not. To date, few if any studies have examined changes in either gluatamatergic or GABAergic plasticity in response to chronic opiate administration. This includes lack of knowledge as to whether there are differences in passive vs. active drug administration, an important consideration given the recent work showing that the persistence of LTP in the VTA of animals abstinent from cocaine self-administration (up to 3 months) occurs only with contingent cocaine exposure (Chen et al. 2008).

However, it is known that chronic morphine, like acute morphine, increases DA neuronal activity. In vivo recordings following chronic morphine show increases in both basal firing rate and burst activity that return to baseline during withdrawal (Georges et al. 2006). This is in contrast to previous work that observed a persistent decrease in DA activity in morphinewithdrawn rats (Diana et al. 1995, 1999). One potential reason for these differences is the administration method used. For example, the Georges et al. study used a subcutaneous (s.c.) sustained release pellet paradigm, which has been shown to have a much different pharmacodynamic profile than the chronic escalating dose paradigm used in the earlier Diana et al. studies. As previously reported (Fischer et al. 2008), $24 \mathrm{hr}$ after the last morphine pellet, blood morphine levels are not decreased, remaining relatively stable with the peak $(\sim 3000 \mathrm{ng} / \mathrm{ml})$, while the chronic injection model produces a much higher peak $(\sim 10,000 \mathrm{ng} / \mathrm{ml})$ at $1 \mathrm{hr}$, with blood levels below $100 \mathrm{ng} / \mathrm{ml}$ after $4 \mathrm{hr}$ and negligible by $12 \mathrm{hr}$. The change in DA firing rate induced by withdrawal from chronic morphine, whether a return to baseline or decrease below baseline, appears to be dependent on changes in GABA release. Withdrawal from chronic morphine increases GABA inhibitory postsynaptic currents (IPSCs) and GABA release onto VTA DA neurons (Bonci and Williams 1997), an effect that has recently been found to be dependent on recycling of the MOR and on cyclic adenosine-5' -monophosphate (cAMP) signaling (Madhavan et al. 2010).

Another potential contributor to differences between studies is the heterogeneity of VTA compared to LC (as described below). Not only is there the complexity of multiple cells types (primarily GABA vs. DA), but the distribution of cell types also varies along the rostral-caudal VTA axis (Fig. 2). Specifically, the proportion of DA to GABA neurons is much higher in rostral VTA subregions (IFN, RL) compared to caudal subregions (PN, PIF) (Nair-Roberts et al. 2008). This difference has functional relevance to morphine-induced behavioral changes. HSV-GluA1 overexpression increased morphine reward behavior with injection into rostral VTA, whereas it induced aversive behavior in caudal VTA, an effect also observed on viral overexpression of cAMP-response-element binding protein (CREB) or phospholipase 


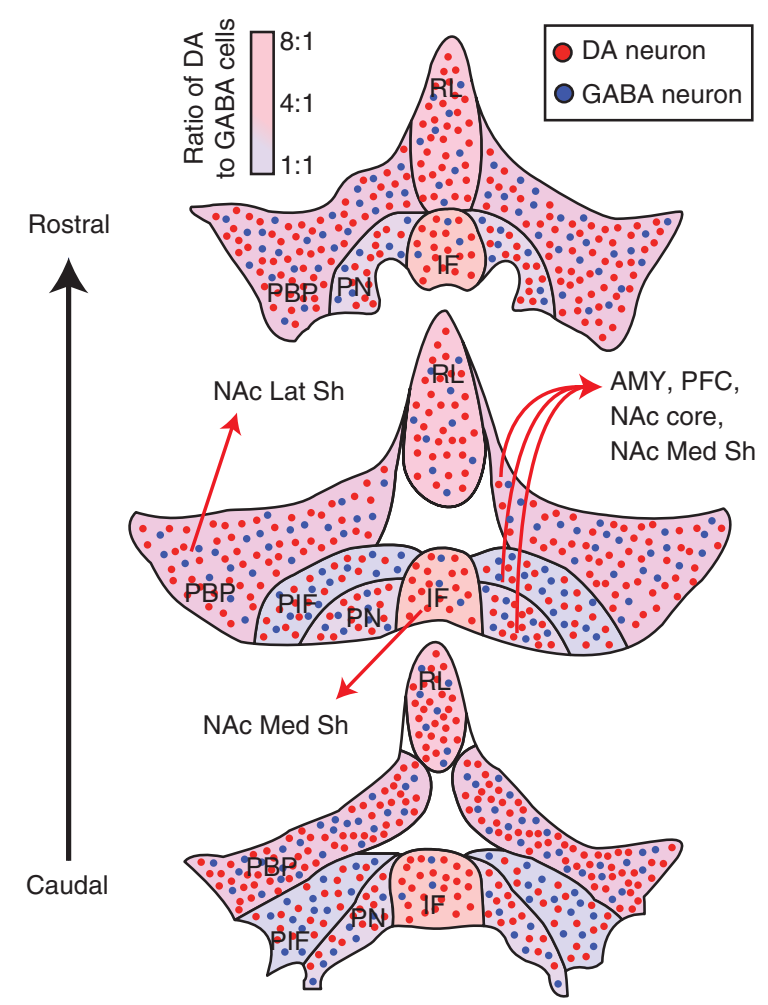

Figure 2. Cellular and projection complexity within VTA. The proportion of DA (red) to GABA (blue) neurons varies among VTA subnuclei with higher DA:GABA ratios observed in more rostral subregions such as rostral linear nucleus (RL) compared to more caudal subnuclei such as paranigral (PN) and parainterfascicular (PIF) regions. Additionally, DA neuronal projections differ throughout with VTA with more lateral regions such as parabrachial nucleus (PBP) projecting to NAc lateral shell (Lat Sh), whereas medial regions such as PN have diverse projections including amygdala (AMY), prefrontal cortex (PFC), NAc core, and NAc medial shell (Med $\mathrm{Sh})$. Limited work has examined GABA neuronal projections; there is some evidence that GABA neurons in rostral PBP have a strong projection to PFC, whereas there are few rostral PBP DA neurons that project to PFC, but a large caudal DA PBP projection; this suggests that the PBP-PFC projection is not only defined regionally, but is also neuronal-subtype specific (Lammel et al. 2008). (Cell counts used are from Nair-Roberts et al. 2008 and projections are from retrograde labeling studies by Lammel et al. 2008.)

C gamma (PLC $\gamma$ ) (Carlezon et al. 2000; Bolanos et al. 2003; Olson et al. 2005). This difference can be seen at the molecular level as well, as chronic morphine induced cAMP response element (CRE)-mediated transcription in DA neurons in rostral and caudal VTA, but was only observed in non-DA neurons in rostral VTA (Olson et al. 2005). Ultrastructural studies confirm such rostral-caudal differences, and suggest the added complexity of treatment regimen and projection output. GluA1 was increased in both tyrosine hydroxylase $(\mathrm{TH})$-positive (DAergic) and TH-negative (likely GABAergic) den- drites in the parabrachial (PBP) VTA with a single morphine injection. In contrast, with chronic morphine, there was an increase in GluA1 in the paranigral (PN) VTA in addition to the PBP region (Lane et al. 2008).

Differences among VTA DA neurons, based on their output region, have been of great interest recently, as it is now well established that the electrophysiological properties of DA neurons vary by projection. VTA DA neurons projecting to NAc have a much smaller $\mathrm{I}_{\mathrm{h}}$ current than neurons projecting to basolateral amygdala (BLA) (Ford et al. 2006), and there are differences in 
projections within NAc itself, with DA neurons projecting to NAc lateral shell displaying much higher $\mathrm{I}_{\mathrm{h}}$ current than DA neurons that project to NAc medial shell (Lammel et al. 2011). Action potential (AP) duration of DA neurons also varies by projection, as NAcprojecting DA neurons have the longest AP duration, while PFC-projecting neuron AP duration is shorter, and AMY-projecting DA neurons have the shortest duration (Margolis et al. 2008). Importantly, responsiveness to opiates also appears to differ within the VTA depending on projection type: DA neurons projecting to NAc responded more to KOR agonists than BLA-projecting neurons, whereas the opposite effect was noted for responsiveness to a MOR/ delta (DOR) agonist, which had a greater effect on BLA-projecting neurons (Ford et al. 2006). This translated to presynaptically-mediated opiate effects as well, as a KOR agonist caused a greater inhibition of $\mathrm{GABA}_{\mathrm{A}}$ IPSCs of DA neurons projecting to BLA, while there was a greater KOR agonist-mediated inhibition of $\mathrm{GABA}_{\mathrm{B}}$ IPSCs in neurons projecting to NAc (Ford et al. 2006). Additionally, it has recently been observed that modulation of excitatory synapses on DA neurons differs depending on projection (Lammel et al. 2011). Lammel and colleagues (2011) found that AMPA/NMDA ratio was increased by cocaine in DA neurons that projected to NAc, but not in DA neurons that projected to PFC. However, AMPA/NMDA ratio was increased in DA cells projecting to PFC in response to an aversive stimulus (hindpaw formalin injection), an effect that was also observed in DA neurons that projected to NAc lateral shell, but absent in DA neurons projecting to NAc medial shell-showing heterogeneity in response within subregions of this projection target (Lammel et al. 2011). Clearly these studies indicate that a more thorough understanding of the synaptic adaptations that occur with both acute and chronic opiates will need to integrate information on the output of the DA neurons studied. The development of neuron- and projection- specific techniques will serve to clarify these issues, by allowing specific modulation in this heterogeneous region.

\section{Opiate-Induced Structural and Cellular Plasticity}

The relevance of drug-induced structural plasticity to synaptic and behavioral changes has been reviewed recently (Russo et al. 2010). Most studies of structural plasticity to date have examined changes in spine morphology or dendritic branching of neurons in VTA target regions, but our laboratory has investigated another structural adaptation in response to chronic opiate administration, an alteration of VTA DA neuron soma size. We first observed that rat VTA DA neuron surface area decreases $\sim 25 \%$ in response to chronic, but not acute, morphine administration (Sklair-Tavron et al. 1996). This effect was specific for DA neurons in VTA, as TH-negative cells (likely GABAergic) were not altered. Additionally, this change could be blocked by systemic naltrexone, suggesting that MOR signaling was required, and local brain-derived neurotrophic factor (BDNF) infusion in VTA also prevented the decrease, suggesting that decreased neurotrophic signaling may underlie the morphological change. Importantly, this reduction in VTA DA neuron soma size is observed with chronic administration of heroin as well as morphine (Russo et al. 2007), in passive and self-administration protocols (Spiga et al. 2003; Chu et al. 2007; Russo et al. 2007), and across species, as we have recently characterized this effect in mouse and in postmortem tissue from human heroin abusers (Mazei-Robison et al. 2011). Follow-up studies found no evidence of VTA DA neuronal death or injury (Sklair-Tavron et al. 1996; Russo et al. 2007) and that the decrease in cell size persists for 14 days after chronic morphine administration, but returns to baseline by 30 days. This time-line mirrors reward tolerance (Russo et al. 2007), in which repeated drug use decreases the rewarding effect of the drug and leads to an escalation of drug intake, as seen in humans (O'Brien 2001).

Given that BDNF could rescue the chronic morphine-induced structural change, we wanted to examine whether downstream neurotrophic signaling pathways mediated this structural plasticity. Although there is some controversy as to whether BDNF levels themselves are altered 
Opiate-Induced VTA and LC Neurons

in VTA in response to chronic opiate administration (Numan et al. 1998; Chu et al. 2007; Koo et al. 2010), regulation has been reported in the three main signaling pathways downstream from BDNF: PLC $\gamma$, phosphatidylinositol 3'-kinase (PI3K), and mitogen-activated protein kinase (MAPK) (Russo et al. 2009). Chronic morphine increases activity of the PLC $\gamma$ pathway (Wolf et al. 1999, 2007), decreases activity of the PI3K pathway, as measured by decreased insulin receptor substrate-2 (IRS2) and phospho-AKT levels (Wolf et al. 1999; Russo et al. 2007; Mazei-Robison et al. 2011), and increases MAPK signaling, as measured by increased phosphorylation and catalytic activity of extracellular-related protein kinase (ERK) (Ortiz et al. 1995; Berhow et al. 1996; Liu et al. 2007). Using viral-mediated overexpression, we found that it was the chronic morphine-induced change in PI3K signaling that contributes to the morphological change: overexpression of a dominant-negative IRS2 (IRS2dn) or AKTdn was sufficient to decrease VTA DA soma size, while overexpression of wild-type IRS2 prevented the morphine-induced decrease and overexpression of a constitutively active AKT (AKTca) increased soma size (Russo et al. 2007; Mazei-Robison et al. 2011). In contrast, overexpression of either PLC $\gamma$ or ERK was not sufficient to alter VTA DA soma size (Russo et al. 2007). Importantly, overexpression of IRS2 was also able to prevent morphine reward tolerance, implicating a role for structural plasticity in behavioral response.

Our recent work suggests that this structural change may be intimately linked to the activity changes induced by chronic opiates. Similar to the in vivo study by Georges et al. discussed above, we found that VTA DA firing rate was increased at the same time-point at which soma size is decreased in mice exposed to chronic morphine (Mazei-Robison et al. 2011). However, we found that DA output to the NAc, as measured by in vivo cyclic voltammetry, is actually decreased, suggesting a break in the normal activation and output in the mesolimbic reward circuit. We further characterized this result and found that IRS2dn overexpression in VTA, which is sufficient to decrease DA soma size, decreased DA output to NAc and also decreased the expression of several $\mathrm{K}^{+}$channel subunits, in a manner similar to chronic morphine. In our efforts to identify the signaling pathways downstream from IRS2/AKT that mediate the chronic morphine-induced neuroadaptations, we made the surprising observation that mammalian target of rapamycin (mTOR) complex 1 (mTORC1) signaling, a well-established pathway in cellular growth, was actually increased by chronic morphine. In contrast, we observed a decrease in mTOR complex 2 (mTORC2) signaling, which we went on to show is both necessary and sufficient for morphine-induced changes in soma size and neuronal activity. Specifically, we found that overexpression of rapamycin-insensitive companion of mTOR (Rictor), an essential component protein of $\mathrm{mTORC} 2$, was sufficient to prevent the decrease in soma size and also prevented the increase in DA neuron firing rate in a cell-autonomous way: only DA cells in VTA that overexpressed Rictor had an attenuated firing rate, whereas nearby DA cells still showed the increase. This suggests that signaling changes intrinsic to DA neurons can mediate excitability changes induced by chronic opiates, possibly by altering AKT modulation of $\mathrm{GABA}_{\mathrm{A}}$ currents (Krishnan et al. 2008) or the expression of $\mathrm{K}^{+}$channels (Mazei-Robison et al. 2011) (Fig. 3). As with IRS2 overexpression, we found that alteration of mTORC2 activity correlated with morphine reward behavior, as decreasing mTORC2 activity decreased morphine conditioned place preference (CPP), while increasing mTORC2 activity was sufficient to induce CPP to a low dose of morphine that does not induce place conditioning in control animals.

It is unlikely that soma size change is the only structural adaptation induced by chronic opiates in the VTA. Given the decreased dendritic spine number and dendritic complexity of branching of NAc medium spiny neurons of rats previously exposed to chronic morphine (Robinson and Kolb 1999; Robinson et al. 2002), we expect that dendritic changes are also occurring in VTA DA neurons. Current studies are underway to characterize spine morphology changes, a huge gap in the field, as only one study to date has examined drug-induced changes in VTA dendritic 
M.S. Mazei-Robison and E.J. Nestler
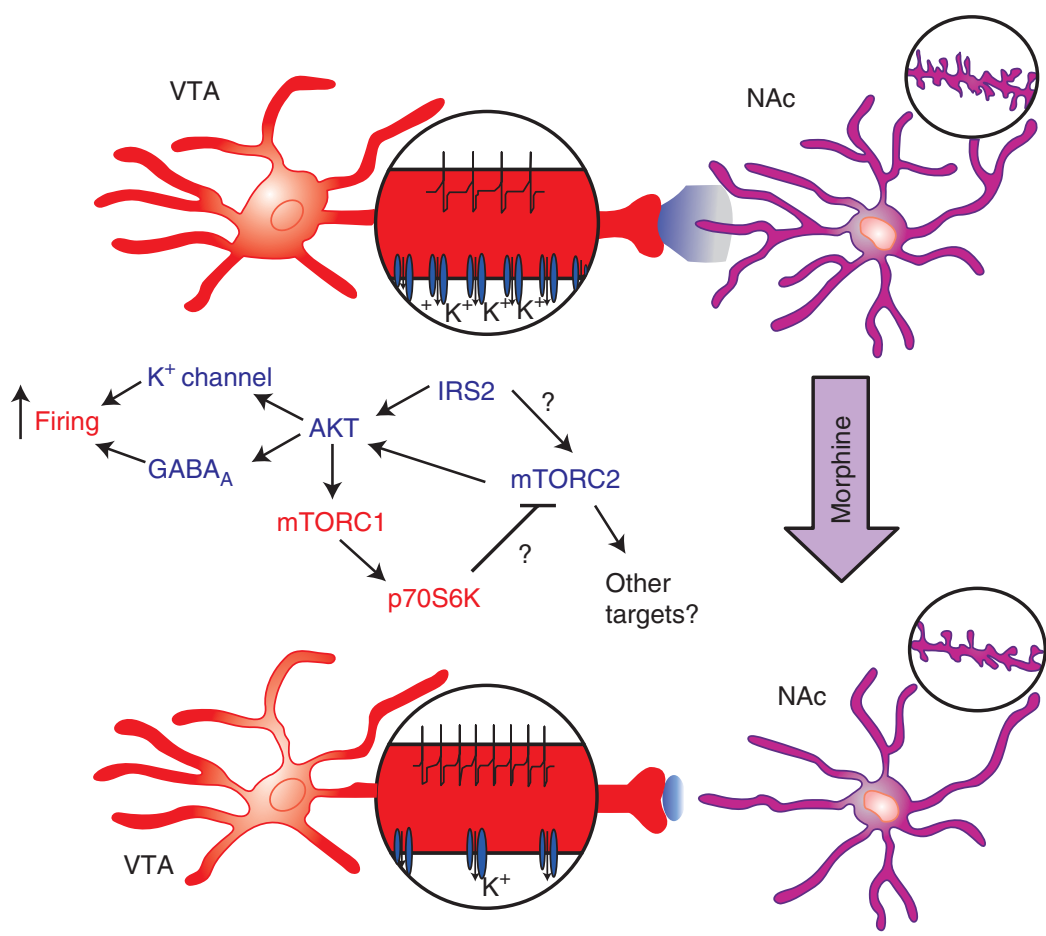

Figure 3. Chronic morphine decreases VTA DA soma size yet increases neuronal excitability, while DA transmission to NAc is decreased. The net effect of morphine is a less responsive reward pathway, i.e., reward tolerance. Down-regulation of IRS2-AKT signaling (blue) in VTA mediates the effects of chronic morphine on soma size and electrical excitability; the effect on excitability is mediated via decreased $\mathrm{GABA}_{\mathrm{A}}$ currents and suppression of $\mathrm{K}^{+}$channel expression. Morphine-induced down-regulation of mTORC2 activity in VTA is crucial for these morphine-induced morphological and physiological adaptations as well as for reward tolerance. In contrast to mTORC2, chronic morphine increases mTORC1 activity (red), which does not appear to directly influence these morphine-induced adaptations. Chronic morphine also decreases DA output to NAc, as well as decreasing dendritic branching and the number of dendritic spines on medium spiny GABA neurons in NAc, further suppressing normal DA signaling in the mesolimbic circuit.

architecture. This study found an increase in dendritic spine density in one subtype of VTA neuron in response to an acute cocaine injection, the same subtype shown to show increased NMDA/AMPA ratio (Sarti et al. 2007). Data from our previous work, that length of VTA DA processes is decreased $(\sim 30 \%)$ in rats treated with chronic morphine (Sklair-Tavron et al. 1996), is consistent with global changes in VTA DA architecture. This change could also help to explain the decrease in DA output to the NAc after chronic morphine, as we have previously reported decreased axonal transport and levels of neurofilament proteins in VTA (Beitner-Johnson et al. 1992, 1993), suggesting that chronic morphine also affects axonal structure and function. Given the regional and projection complexity in VTA DA neurons noted above, we are currently examining whether these structural changes are induced in a particular subset of VTA DA neurons using fluorescent retrograde tracers. These data will be critical to understanding the structural and electrophysiological changes induced by chronic opiates and the relevant output circuits involved.

As alluded to earlier, several studies, both molecular and electrophysiological, have provided evidence that chronic opiate administration activates the cAMP-CREB pathway in the VTA 
Opiate-Induced VTA and LC Neurons

(Bonci and Williams 1997; Olson et al. 2005; Madhavan et al. 2010). Also, a microarray study defined the global changes in gene expression that occur in VTA in response to chronic morphine (McClung et al. 2005). Work is now needed to better define the cellular specificity of these neuroadaptations as well as delineate their functional consequences. Moreover, while most work on VTA has focused on opiate-induced neuroadaptations presumed to occur in DA neurons, it is essential to explore drug-induced plasticity that occurs in the VTA's GABAergic neurons, which are one of the key initial targets of opiate action in this brain region.

\section{LOCUS COERULEUS}

\section{Background}

The LC is the main site of norepinephrine (NE)containing neurons in the brain (Dahlstrom and Fuxe 1965). As reviewed previously (Aston-Jones and Bloom 1981a; Aston-Jones et al. 1991b; Berridge and Waterhouse 2003; Van Bockstaele et al. 2010), LC is a discrete, compact, homogeneous nucleus, consisting of almost exclusively NE neurons. The major inputs to LC are from the medullary nucleus paragigantocellularis (PGi) and nucleus prepositus hypoglossus, and LC outputs are widespread including forebrain, cerebellum, brainstem, and spinal cord (Fig. 1) (Berridge and Waterhouse 2003). LC neuronal activity is highly synchronous both basally and in response to stimuli (Foote et al. 1980; Aston-Jones and Bloom 1981b; Aston-Jones et al. 1991a; Ishimatsu and Williams 1996). LC neurons are spontaneously active (Williams et al. 1991) and their activation elicits NE release in several forebrain regions including cortex and hippocampus. The LC largely serves as a relay nucleus, with limited synaptic plasticity noted to date, although glutamate afferents control LC activity, notably from PGi (Ennis et al. 1992). LC neurons express the three main classes of opioid receptors: MOR, DOR, and KOR with distinct distribution, although, as with the VTA, our discussion is limited to the MOR, which is most directly implicated in opiate dependence and addiction.

\section{Opiate-Induced Cellular Plasticity}

Although there is no evidence of traditional synaptic plasticity (i.e., LTP and LTD) in LC, there is well-described cellular plasticity. A unique feature of LC is that many of its in vivo responses to chronic opiates can be recapitulated and studied at the single-cell level (Nestler et al. 1994; Nestler and Aghajanian 1997; Nestler 2004). Binding of opiates (e.g., morphine) to the MOR leads to decreased adenylyl cyclase (AC) activity and cAMP signaling (Duman et al. 1988). Acute binding of opiates to the MOR also decreases the pacemaker activity of LC neurons, largely by activating $\mathrm{G}$ protein-gated inwardly-rectifying $\mathrm{K}^{+}$(GIRK) channels (Williams et al. 1982; Torrecilla et al. 2002). However, with chronic opiate administration, both the firing rate and cAMP signaling return to baseline because of an up-regulation of the cAMP pathway, illustrating tolerance (Aghajanian 1978; Duman et al. 1988; Nestler and Tallman 1988; Guitart and Nestler 1989; Kogan et al. 1992; Ivanov and Aston-Jones 2001). This plasticity induced by chronic opiate administration (i.e., cAMP pathway up-regulation) becomes functionally evident on withdrawal of the opiate, when the firing rate of LC neurons is significantly increased along with a large increase in cAMP activity, illustrating dependence and withdrawal (Fig. 4) (Aghajanian 1978; Rasmussen et al. 1990).

These adaptations are mediated via the upregulation of several signaling proteins in the cAMP pathway including AC1/8 (Matsuoka et al. 1994; Lane-Ladd et al. 1997; Zachariou et al. 2008), cAMP-dependent protein kinase (PKA) (Nestler and Tallman 1988), CREB (Guitart et al. 1992; Shaw-Lutchman et al. 2002; Han et al. 2006), and TH and BDNF-both downstream CREB targets (Guitart et al. 1989; Akbarian et al. 2002). Chronic opiates also induce GIRK2/3 expression in LC (Cruz et al. 2008) as well as numerous other genes as revealed by microarray analysis (McClung et al. 2005). Furthermore, it has recently been shown, using an LC slice culture model, that the increased intrinsic electrical activity of LC neurons induced by chronic opiates is caused by the direct activation of MOR on LC NE neurons, implicating an 
M.S. Mazei-Robison and E.J. Nestler
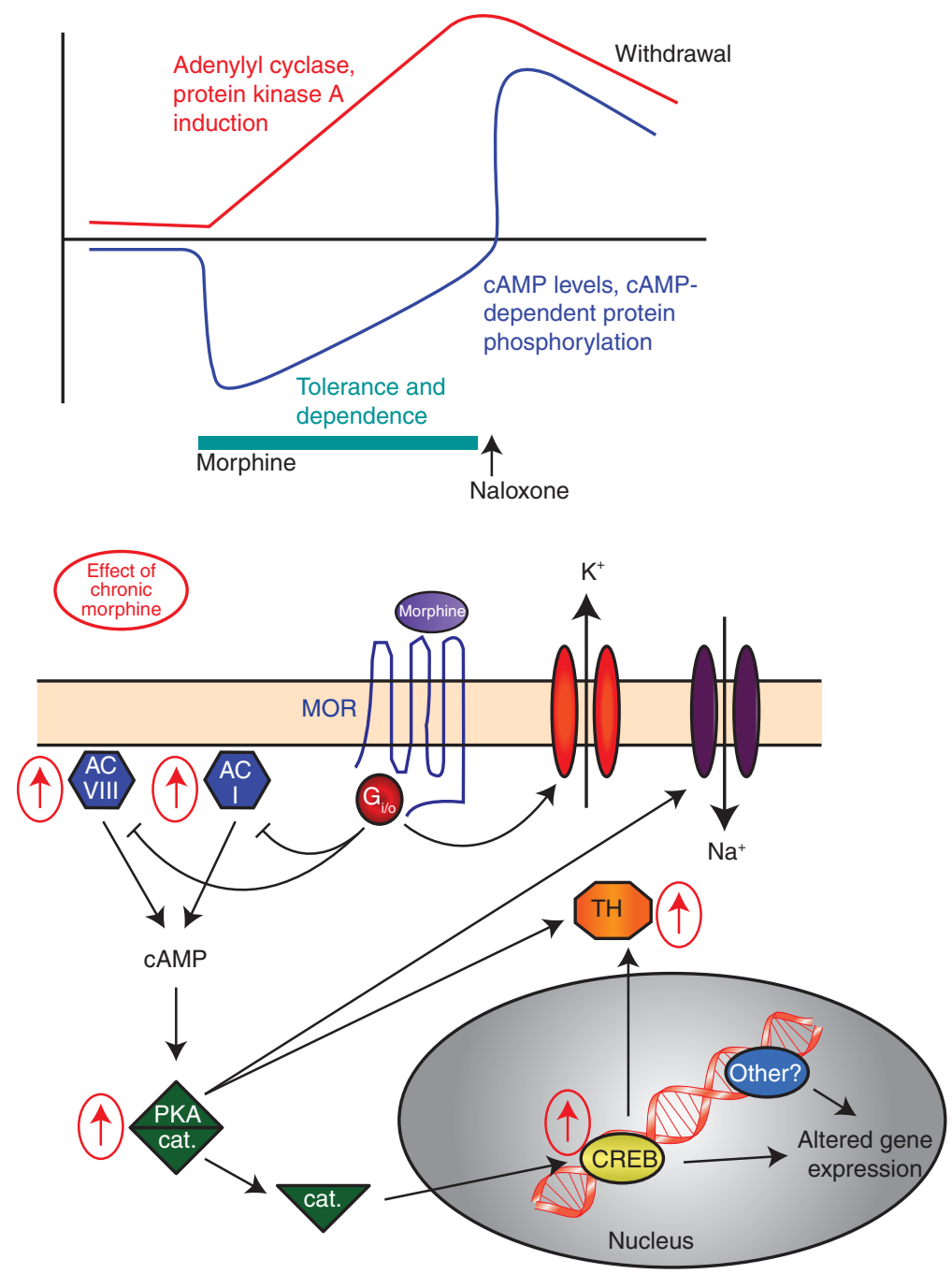

Figure 4. Up-regulation of the cAMP pathway in LC as a mechanism of opiate tolerance and dependence. Top panel, Opiates acutely inhibit the functional activity of the cAMP pathway (indicated by cellular levels of cAMP and cAMP-dependent protein phosphorylation). With continued opiate exposure, functional activity of the cAMP pathway gradually recovers, and increases far above control levels following removal of the opiate (e.g., by administration of the opioid receptor antagonist naloxone). These changes in the functional state of the cAMP pathway are mediated via induction of adenylyl cyclases (AC) and protein kinase A (PKA) in response to chronic administration of opiates. Induction of these enzymes accounts for the gradual recovery in the functional activity of the cAMP pathway that occurs during chronic opiate exposure (tolerance and dependence) and activation of the cAMP pathway observed on removal of opiate (withdrawal). Bottom panel, Opiates acutely inhibit LC neurons by increasing the conductance of an inwardly rectifying $\mathrm{K}^{+}$channel via coupling with subtypes of $\mathrm{G}_{\mathrm{i} / \mathrm{o}}$ and, possibly, by decreasing a $\mathrm{Na}^{+}$-dependent inward current via coupling with $\mathrm{G}_{\mathrm{i} / \mathrm{o}}$ and the consequent inhibition of AC, reduced levels of PKA activity, and reduced phosphorylation of the channel or pump responsible. Inhibition of the cAMP pathway also decreases the phosphorylation of many other proteins and, thereby, affects numerous other neuronal processes. For example, it reduces the phosphorylation state of cAMP response element-binding protein (CREB), which initiates some of the longer-term changes in LC function. Chronic administration of morphine increases the levels of ACI, ACVIII, PKA catalytic (cat.) and regulatory subunits, and several phosphoproteins, including CREB and tyrosine hydroxylase (TH) (indicated by red arrows). (Legend continues on facing page.) 
intrinsic homeostatic adaptation (Cao et al. 2010). This approach identified a crucial role for CREB in both the pacemaker activity and morphine-induced increase in LC firing rate (Han et al. 2006; Cao et al. 2010), an effect that was also observed in mice with an early developmental knockout of CREB specific to NE neurons (Parlato et al. 2010). Finally, this activation of LC neuronal firing, and the up-regulated cAMP-CREB pathway, which mediates the increased firing, have been shown in numerous studies to be both necessary and sufficient to mediate several symptoms of physical opiate withdrawal (Lane-Ladd et al. 1997; Punch et al. 1997; Han et al. 2006).

Although most of the opiate-induced plasticity described here is postulated to be intrinsic to LC NE neurons, there is some evidence that chronic morphine can also influence excitatory input to LC as there is an increase in spontaneous EPSC frequency in slices from morphinetreated mice (Torrecilla et al. 2008). Additionally, there is an increase in glutamate and aspartate release in LC in vivo in morphine-withdrawn rats and local application of excitatory amino acid antagonists in LC partially blocks the withdrawal-induced increase in LC activity (Akaoka and Aston-Jones 1991; Aghajanian et al. 1994).

Some controversy remains as to whether the changes in cAMP-CREB signaling in LC neurons and in LC neuronal activity mediate opiate withdrawal behaviors. For example, lesions of LC, or developmental knockout of CREB activity in LC NE neurons, fail to detectably alter withdrawal symptoms (Christie et al. 1997; Parlato et al. 2010). In contrast, we have shown that modulation of the activity of the cAMP pathway or of CREB in LC of adult animals consistently blocks several withdrawal behaviors (Lane-Ladd et al. 1997; Punch et al. 1997; Han et al. 2006).
We believe that several key considerations explain these differing findings. First, LC is just one of several brain areas important for physical opiate dependence and withdrawal (Koob and Le Moal 2001). It is not surprising that animals with lesioned LCs still develop profound physical dependence mediated by increased reliance on these other neural substrates. Second, it is very plausible that some of the tools used to manipulate cAMP pathway activity in LC (e.g., local infusion of PKA activators or inhibitors) influence glutamatergic afferents in this region, which also appear to show plastic changes (including cAMP pathway up-regulation) after chronic morphine (Nestler 1992; Christie et al. 1997). Third, despite a likely role for these glutamatergic afferents, there is no question that plasticity intrinsic to LC NE neurons is also involved, because local knockout of CREB from the adult LC (which cannot affect afferent nerve terminals) blocks the morphine-induced increased excitability of LC NE neurons and attenuates withdrawal (Cao et al. 2010; V Zachariou and EJ Nestler, unpubl.). The lack of effect of CREB knockout from these neurons in conditional knockout mice (Parlato et al. 2010) highlights the developmental compensations that complicate the use of early knockout models and emphasizes the importance of using gene manipulations in the fully differentiated adult brain when studying adult plasticity.

Thus, a wealth of experimental evidence establishes up-regulation of the CAMP-CREB pathway as a mechanism of intrinsic homeostatic plasticity in LC NE neurons in the development of opiate physical dependence. It is also important to emphasize the historical importance of this work on LC, as it served as a model system for the long-term actions of opiates on the brain: based on these earlier investigations of LC, up-regulation of the CAMP-CREB

Figure 4. (Continued) These changes contribute to the altered phenotype of the drug-addicted state. For example, the intrinsic excitability of LC neurons is increased by enhanced activity of the cAMP pathway and $\mathrm{Na}^{+}$-dependent inward current, which contributes to the tolerance, dependence, and withdrawal showed by these neurons. Up-regulation of ACVIII and TH is mediated via CREB, whereas up-regulation of ACI and of the PKA subunits appears to occur via an unidentified, CREB-independent mechanism. 
M.S. Mazei-Robison and E.J. Nestler

pathway has since been shown to be a common mechanism of opiate tolerance, dependence, and withdrawal in numerous regions of the central and peripheral nervous systems and indeed represents one of the best established models of the molecular basis of drug addiction (Nestler 2001, 2004).

\section{OPIATE-INDUCED STRUCTURAL PLASTICITY}

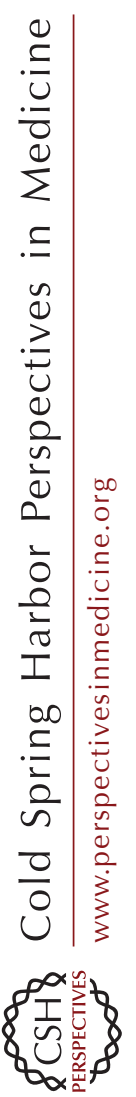

To date, there has not been a description of structural plasticity in LC neurons in response to chronic opiate administration. We are currently assessing whether any soma size changes occur in these neurons analogous to the changes observed in DA neurons in VTA. However, two lines of evidence suggest that this type of change may not be relevant in LC. First, normal axonal transport and levels of neurofilament proteins were observed in LC after chronic morphine in contrast to VTA (Beitner-Johnson et al. 1992; Beitner-Johnson and Nestler 1993), suggesting that trophic support of neuronal structure may not be affected. Second, given our finding that increased firing rate is a key contributor to changes in soma size, the differences between opiate regulation of firing rates in LC and VTA may be important. Namely, in VTA, opiates acutely and chronically increase firing rate in slices and in vivo, and we observe a decreased cell size coincident with and as a consequence of this increase in firing rate. This increased rate then normalizes, or even decreases below baseline, in animals withdrawn from the opiate. Because there is evidence from our own work (Russo et al. 2007), and others (Spiga et al. 2003), that the soma size is also decreased at these later time-points, when firing rate has decreased, it may be the initial sustained increase in firing rate that is vital for induction or maintenance of the morphological change. In contrast, LC neuronal activity is acutely decreased by morphine administration, returns toward baseline levels in vivo with chronic administration, and only increases above normal levels on opiate withdrawal. (These in vivo observations differ from what occurs in brain slice cultures, in which the increased firing rate and CAMP-CREB pathway up-regulation occur in the chronic morphine-treated [dependent] state, without withdrawal [Cao et al. 2010].) These considerations suggest that, whereas chronic morphine may not elicit a change in structural plasticity in LC neurons in vivo, withdrawal from morphine might. In support of this idea, results from our microarray study of LC found that several genes involved in cell growth and structure are decreased or unchanged with chronic morphine, but are increased with withdrawal (McClung et al. 2005). It is known that prolonged decreases in the basal firing rate of LC neurons is not sufficient to alter soma size, as early CREB knockout from LC NE neurons did not alter neuronal size but decreased basal activity (Parlato et al. 2010). However, we also did not detect a difference in VTA DA soma size when we overexpressed a $\mathrm{K}^{+}$channel to decrease firing rate (Mazei-Robison et al. 2011), so the Parlato et al. observations do not preclude the possibility of a morphine withdrawal-induced change. Still, it should be noted that the mechanism mediating the changes in firing rate between the two brain regions is very different, with changes in AKT signaling, $\mathrm{GABA}_{\mathrm{A}}$ currents, and $\mathrm{K}^{+}$channel expression implicated in VTA and CAMP-CREB signaling implicated in LC.

\section{CONCLUDING REMARKS}

Together, data from VTA and LC illustrate the complex and important changes in synaptic, cellular, and structural plasticity that mediate the lasting effects of opiate drugs on the brain's catecholamine neurons and other neuronal types in these regions, which in turn influence drug reward and dependence. Although the plasticity that underlies acute opiate action in both regions, and chronic opiate action in LC, is fairly well characterized, future studies are needed to delineate the plasticity that occurs with chronic opiate administration in VTA with respect to differences seen across multiple cell types and across multiple input-output patterns even for a single cell type. Such advances will contribute to a better understanding of how opiates influence this brain region to control reward and ultimately addiction. Such an understanding of 
Opiate-Induced VTA and LC Neurons

the long-lasting adaptations induced by opiates in VTA and LC will improve not only our knowledge of the etiology of opiate dependence and addiction, but will also help us elucidate novel therapeutic interventions.

\section{ACKNOWLEDGMENTS}

We would like to thank A.J. Robison and Jessica Ables for artistic assistance.

\section{REFERENCES}

Aghajanian GK. 1978. Tolerance of locus coeruleus neurones to morphine and suppression of withdrawal response by clonidine. Nature 276: 186-188.

Aghajanian GK, Kogan JH, Moghaddam B. 1994. Opiate withdrawal increases glutamate and aspartate efflux in the locus coeruleus: An in vivo microdialysis study. Brain Res 636: $126-130$

Akaoka H, Aston-Jones G. 1991. Opiate withdrawal-induced hyperactivity of locus coeruleus neurons is substantially mediated by augmented excitatory amino acid input. J Neurosci 11: 3830-3839.

Akbarian S, Rios M, Liu RJ, Gold SJ, Fong HF, Zeiler S, Coppola V, Tessarollo L, Jones KR, Nestler EJ, et al. 2002. Brain-derived neurotrophic factor is essential for opiate-induced plasticity of noradrenergic neurons. $J$ Neurosci 22: 4153-4162.

Aston-Jones G, Bloom FE. 1981a. Activity of norepinephrine-containing locus coeruleus neurons in behaving rats anticipates fluctuations in the sleep-waking cycle. J Neurosci 1: 876-886.

Aston-Jones G, Bloom FE. 1981b. Norepinephrine-containing locus coeruleus neurons in behaving rats exhibit pronounced responses to non-noxious environmental stimuli. J Neurosci 1: 887-900.

Aston-Jones G, Chiang C, Alexinsky T. 1991a. Discharge of noradrenergic locus coeruleus neurons in behaving rats and monkeys suggests a role in vigilance. Prog Brain Res 88: $501-520$.

Aston-Jones G, Shipley MT, Chouvet G, Ennis M, van Bockstaele E, Pieribone V, Shiekhattar R, Akaoka H, Drolet G, Astier B, et al. 1991b. Afferent regulation of locus coeruleus neurons: Anatomy, physiology and pharmacology. Prog Brain Res 88: 47-75.

Ballantyne JC, LaForge KS. 2007. Opioid dependence and addiction during opioid treatment of chronic pain. Pain 129: 235-255.

Beitner-Johnson D, Nestler EJ. 1993. Chronic morphine impairs axoplasmic transport in the rat mesolimbic dopamine system. Neuroreport 5: 57-60.

Beitner-Johnson D, Guitart X, Nestler EJ. 1992. Neurofilament proteins and the mesolimbic dopamine system: Common regulation by chronic morphine and chronic cocaine in the rat ventral tegmental area. J Neurosci 12: 2165-2176.
Berhow MT, Hiroi N, Nestler EJ. 1996. Regulation of ERK (extracellular signal regulated kinase), part of the neurotrophin signal transduction cascade, in the rat mesolimbic dopamine system by chronic exposure to morphine or cocaine. J Neurosci 16: 4707-4715.

Berridge CW, Waterhouse BD. 2003. The locus coeruleusnoradrenergic system: Modulation of behavioral state and state-dependent cognitive processes. Brain Res Brain Res Rev 42: 33-84.

Bolanos CA, Perrotti LI, Edwards S, Eisch AJ, Barrot M, Olson VG, Russell DS, Neve RL, Nestler EJ. 2003. Phospholipase Cgamma in distinct regions of the ventral tegmental area differentially modulates mood-related behaviors. J Neurosci 23: 7569-7576.

Bonci A, Williams JT. 1997. Increased probability of GABA release during withdrawal from morphine. J Neurosci 17: 796-803.

Brown MT, Bellone C, Mameli M, Labouebe G, Bocklisch C, Balland B, Dahan L, Lujan R, Deisseroth K, Luscher C. 2010. Drug-driven AMPA receptor redistribution mimicked by selective dopamine neuron stimulation. PLoS ONE 5: e15870.

Cao JL, Vialou VF, Lobo MK, Robison AJ, Neve RL, Cooper DC, Nestler EJ, Han MH. 2010. Essential role of the cAMP-cAMP response-element binding protein pathway in opiate-induced homeostatic adaptations of locus coeruleus neurons. Proc Natl Acad Sci 107: 17011-17016.

Carlezon WA Jr, Boundy VA, Haile CN, Lane SB, Kalb RG, Neve RL, Nestler EJ. 1997. Sensitization to morphine induced by viral-mediated gene transfer. Science 277: 812-814.

Carlezon WA Jr, Haile CN, Coppersmith R, Hayashi Y, Malinow R, Neve RL, Nestler EJ. 2000. Distinct sites of opiate reward and aversion within the midbrain identified using a herpes simplex virus vector expressing GluR1. J Neurosci 20: RC62.

Chen BT, Bowers MS, Martin M, Hopf FW, Guillory AM, Carelli RM, Chou JK, Bonci A. 2008. Cocaine but not natural reward self-administration nor passive cocaine infusion produces persistent LTP in the VTA. Neuron 59: $288-297$.

Christie MJ, Williams JT, Osborne PB, Bellchambers CE. 1997. Where is the locus in opioid withdrawal? Trends Pharmacol Sci 18: 134-140.

Chu NN, Zuo YF, Meng L, Lee DY, Han JS, Cui CL. 2007. Peripheral electrical stimulation reversed the cell size reduction and increased BDNF level in the ventral tegmental area in chronic morphine-treated rats. Brain Res 1182C: 90-98.

Compton WM, Volkow ND. 2006. Major increases in opioid analgesic abuse in the United States: Concerns and strategies. Drug Alcohol Depend 81: 103-107.

Cruz HG, Berton F, Sollini M, Blanchet C, Pravetoni M, Wickman K, Luscher C. 2008. Absence and rescue of morphine withdrawal in GIRK/Kir3 knock-out mice. J Neurosci 28: 4069-4077.

Dacher M, Nugent FS. 2011a. Morphine-induced modulation of LTD at GABAergic synapses in the ventral tegmental area. Neuropharmacology 61: 1166-1171.

Dacher M, Nugent FS. 2011b. Opiates and plasticity. Neuropharmacology 61: 1088-1096. 
M.S. Mazei-Robison and E.J. Nestler

Dahlstrom A, Fuxe K. 1965. Evidence for the existence of an outflow of noradrenaline nerve fibres in the ventral roots of the rat spinal cord. Experientia 21: 409-410.

Diana M, Pistis M, Muntoni A, Gessa G. 1995. Profound decrease of mesolimbic dopaminergic neuronal activity in morphine withdrawn rats. J Pharmacol Exp Ther 272: $781-785$.

Diana M, Muntoni AL, Pistis M, Melis M, Gessa GL. 1999. Lasting reduction in mesolimbic dopamine neuronal activity after morphine withdrawal. Eur J Neurosci 11: 1037-1041.

Di Chiara G, Imperato A. 1988. Drugs abused by humans preferentially increase synaptic dopamine concentrations in the mesolimbic system of freely moving rats. Proc Natl Acad Sci 85: 5274-5278.

Duman RS, Tallman JF, Nestler EJ. 1988. Acute and chronic opiate-regulation of adenylate cyclase in brain: Specific effects in locus coeruleus. J Pharmacol Exp Ther 246: 1033-1039.

Ennis M, Aston-Jones G, Shiekhattar R. 1992. Activation of locus coeruleus neurons by nucleus paragigantocellularis or noxious sensory stimulation is mediated by intracoerulear excitatory amino acid neurotransmission. Brain Res 598: 185-195.

Fields HL. 2011. The doctor's dilemma: Opiate analgesics and chronic pain. Neuron 69: 591-594.

Fischer SJ, Arguello AA, Charlton JJ, Fuller DC, Zachariou V, Eisch AJ. 2008. Morphine blood levels, dependence, and regulation of hippocampal subgranular zone proliferation rely on administration paradigm. Neuroscience 151: 1217-1224.

Foote SL, Aston-Jones G, Bloom FE. 1980. Impulse activity of locus coeruleus neurons in awake rats and monkeys is a function of sensory stimulation and arousal. Proc Natl Acad Sci 77: 3033-3037.

Ford CP, Mark GP, Williams JT. 2006. Properties and opioid inhibition of mesolimbic dopamine neurons vary according to target location. J Neurosci 26: 2788-2797.

Geisler S, Derst C, Veh RW, Zahm DS. 2007. Glutamatergic afferents of the ventral tegmental area in the rat. J Neurosci 27: $5730-5743$.

Georges F, Le Moine C, Aston-Jones G. 2006. No effect of morphine on ventral tegmental dopamine neurons during withdrawal. J Neurosci 26: 5720-5726.

Guitart X, Nestler EJ. 1989. Identification of morphine- and cyclic AMP-regulated phosphoproteins (MARPPs) in the locus coeruleus and other regions of rat brain: Regulation by acute and chronic morphine. J Neurosci 9: 4371-4387.

Guitart X, Thompson MA, Mirante CK, Greenberg ME, Nestler EJ. 1992. Regulation of cyclic AMP response element-binding protein (CREB) phosphorylation by acute and chronic morphine in the rat locus coeruleus. J Neurochem 58: 1168-1171.

Gysling K, Wang RY. 1983. Morphine-induced activation of A10 dopamine neurons in the rat. Brain Res 277: 119127.

Han MH, Bolanos CA, Green TA, Olson VG, Neve RL, Liu RJ, Aghajanian GK, Nestler EJ. 2006. Role of cAMP response element-binding protein in the rat locus ceruleus: Regulation of neuronal activity and opiate withdrawal behaviors. J Neurosci 26: 4624-4629.
Ishimatsu M, Williams JT. 1996. Synchronous activity in locus coeruleus results from dendritic interactions in pericoerulear regions. J Neurosci 16: 5196-5204.

Ivanov A, Aston-Jones G. 2001. Local opiate withdrawal in locus coeruleus neurons in vitro. J Neurophysiol 85: 2388-2397.

Johnson SW, North RA. 1992. Opioids excite dopamine neurons by hyperpolarization of local interneurons. $J$ Neurosci 12: 483-488.

Kogan JH, Nestler EJ, Aghajanian GK. 1992. Elevated basal firing rates of locus coeruleus neurons in brain slices from opiate-dependent rats: Association with enhanced responses to 8-Br-cAMP. Eur J Pharmacol 211: 47-53.

Koo JW, Mazei-Robison MS, Laplant Q, Dietz DM, Ferguson D, Lobo M, Ohnishi YN, Feng J, Ohnishi YH, Mouzon E, et al. 2010. Role of BDNF in the VTA in regulating molecular and behavioral responses to morphine. In 40th Annual Meeting, Neuroscience 2010, \#368.5, Society for Neuroscience, Washington, D.C.

Koob GF, Le Moal M. 2001. Drug addiction, dysregulation of reward, and allostasis. Neuropsychopharmacology 24: 97-129.

Krishnan V, Han MH, Mazei-Robison M, Iniguez SD, Ables JL, Vialou V, Berton O, Ghose S, Covington HE 3rd, Wiley MD, et al. 2008. AKT signaling within the ventral tegmental area regulates cellular and behavioral responses to stressful stimuli. Biol Psychiatry 64: 691-700.

Kuehn BM. 2007. Opioid prescriptions soar: Increase in legitimate use as well as abuse. JAMA 297: 249-251.

Lammel S, Hetzel A, Häckel O, Jones I, Liss B, Roeper J. 2008. Unique properties of mesoprefrontal neurons within a dual mesocorticolimbic dopamine system. Neuron 57: 760-773.

Lammel S, Ion DI, Roeper J, Malenka RC. 2011. Projectionspecific modulation of dopamine neuron synapses by aversive and rewarding stimuli. Neuron 70: 855-862.

Lane DA, Lessard AA, Chan J, Colago EE, Zhou Y, Schlussman SD, Kreek MJ, Pickel VM. 2008. Region-specific changes in the subcellular distribution of AMPA receptor GluR1 subunit in the rat ventral tegmental area after acute or chronic morphine administration. J Neurosci 28: 9670-9681.

Lane-Ladd SB, Pineda J, Boundy VA, Pfeuffer T, Krupinski J, Aghajanian GK, Nestler EJ. 1997. CREB (cAMP response element-binding protein) in the locus coeruleus: Biochemical, physiological, and behavioral evidence for a role in opiate dependence. J Neurosci 17: 7890-7901.

Leone P, Pocock D, Wise RA. 1991. Morphine-dopamine interaction: Ventral tegmental morphine increases nucleus accumbens dopamine release. Pharmacol Biochem Behav 39: 469-472.

Liu Y, Wang Y, Jiang Z, Wan C, Zhou W, Wang Z. 2007. The extracellular signal-regulated kinase signaling pathway is involved in the modulation of morphine-induced reward by mPer1. Neuroscience 146: 265-271.

Luscher C, Malenka RC. 2011. Drug-evoked synaptic plasticity in addiction: From molecular changes to circuit remodeling. Neuron 69: 650-663.

Madhavan A, He L, Stuber GD, Bonci A, Whistler JL. 2010. micro-Opioid receptor endocytosis prevents adaptations in ventral tegmental area GABA transmission induced 
Opiate-Induced VTA and LC Neurons

during naloxone-precipitated morphine withdrawal. $J$ Neurosci 30: 3276-3286.

Manchikanti L, Fellows B, Ailinani H, Pampati V. 2010. Therapeutic use, abuse, and nonmedical use of opioids: A ten-year perspective. Pain Physician 13: 401-435.

Margolis EB, Hjelmstad GO, Bonci A, Fields HL. 2003. Kappa-opioid agonists directly inhibit midbrain dopaminergic neurons. J Neurosci 23: 9981-9986.

Margolis EB, Lock H, Hjelmstad GO, Fields HL. 2006. The ventral tegmental area revisited: Is there an electrophysiological marker for dopaminergic neurons? J Physiol 577: 907-924.

Margolis EB, Mitchell JM, Ishikawa J, Hjelmstad GO, Fields HL. 2008. Midbrain dopamine neurons: Projection target determines action potential duration and dopamine D(2) receptor inhibition. J Neurosci 28: 8908-8913.

Matsuoka I, Maldonado R, Defer N, Noel F, Hanoune J, Roques BP. 1994. Chronic morphine administration causes region-specific increase of brain type VIII adenylyl cyclase mRNA. Eur J Pharmacol 268: 215-221.

Mazei-Robison MS, Koo JW, Friedman AK, Lansink CS, Robison AJ, Vinish M, Krishnan V, Kim S, Siuta MA, Galli A, et al. 2011. Role for mTOR signaling and neuronal activity in morphine-induced adaptations in ventral tegmental area dopamine neurons. Neuron 72: 977-990.

McClung CA, Nestler EJ, Zachariou V. 2005. Regulation of gene expression by chronic morphine and morphine withdrawal in the locus ceruleus and ventral tegmental area. J Neurosci 25: 6005-6015.

Nair-Roberts RG, Chatelain-Badie SD, Benson E, WhiteCooper H, Bolam JP, Ungless MA. 2008. Stereological estimates of dopaminergic, GABAergic and glutamatergic neurons in the ventral tegmental area, substantia nigra and retrorubral field in the rat. Neuroscience 152: 1024 1031.

Nestler EJ. 1992. Molecular mechanisms of drug addiction. J Neurosci 12: 2439-2450.

Nestler EJ. 2001. Molecular basis of long-term plasticity underlying addiction. Nature Rev Neurosci 2: 119-128.

Nestler EJ. 2004. Historical review: Molecular and cellular mechanisms of opiate and cocaine addiction. Trends Pharmacol Sci 25: 210-218.

Nestler EJ, Aghajanian GK. 1997. Molecular and cellular basis of addiction. Science 278: 58-63.

Nestler EJ, Tallman JF. 1988. Chronic morphine treatment increases cyclic AMP-dependent protein kinase activity in the rat locus coeruleus. Mol Pharmacol 33: 127-132.

Nestler EJ, Alreja M, Aghajanian GK. 1994. Molecular and cellular mechanisms of opiate action: Studies in the rat locus coeruleus. Brain Res Bull 35: 521-528.

Niehaus JL, Murali M, Kauer JA. 2010. Drugs of abuse and stress impair LTP at inhibitory synapses in the ventral tegmental area. Eur J Neurosci 32: 108-117.

Nugent FS, Penick EC, Kauer JA. 2007. Opioids block longterm potentiation of inhibitory synapses. Nature 446: 1086-1090.

Nugent FS, Niehaus JL, Kauer JA. 2009. PKG and PKA signaling in LTP at GABAergic synapses. Neuropsychopharmacology 34: 1829-1842.

Numan S, Lane-Ladd SB, Zhang L, Lundgren KH, Russell DS, Seroogy KB, Nestler EJ. 1998. Differential regulation of neurotrophin and trk receptor mRNAs in catecholaminergic nuclei during chronic opiate treatment and withdrawal. J Neurosci 18: 10700-10708.

O'Brien CP. 2001. Drug addiction and drug abuse. In Goodman and Gilman's The pharmacological basis of therapeutics (ed. Hardman JG, Limbird LE, Gilman AG), pp. 621-642. McGraw-Hill, New York.

Olson VG, Zabetian CP, Bolanos CA, Edwards S, Barrot M, Eisch AJ, Hughes T, Self DW, Neve RL, Nestler EJ. 2005. Regulation of drug reward by cAMP response elementbinding protein: Evidence for two functionally distinct subregions of the ventral tegmental area. J Neurosci 25: $5553-5562$.

Ortiz J, Harris HW, Guitart X, Terwilliger RZ, Haycock JW, Nestler EJ. 1995. Extracellular signal-regulated protein kinases (ERKs) and ERK kinase (MEK) in brain: Regional distribution and regulation by chronic morphine. J Neurosci 15: 1285-1297.

Parlato R, Cruz H, Otto C, Murtra P, Parkitna JR, Martin M, Bura SA, Begus-Nahrmann Y, von Bohlen und Halbach O, Maldonado R, et al. 2010. Effects of the cell typespecific ablation of the cAMP-responsive transcription factor in noradrenergic neurons on locus coeruleus firing and withdrawal behavior after chronic exposure to morphine. J Neurochem 115: 563-573.

Punch L, Self DW, Nestler EJ, Taylor JR. 1997. Opposite modulation of opiate withdrawal behaviors upon microinfusion of a protein kinase A inhibitor versus activator into the locus coeruleus or periaqueductal gray. J Neurosci 17: 8520-8527.

Rasmussen K, Beitner-Johnson DB, Krystal JH, Aghajanian GK, Nestler EJ. 1990. Opiate withdrawal and the rat locus coeruleus: Behavioral, electrophysiological, and biochemical correlates. J Neurosci 10: 2308-2317.

Robinson TE, Kolb B. 1999. Morphine alters the structure of neurons in the nucleus accumbens and neocortex of rats. Synapse 33: 160-162.

Robinson TE, Gorny G, Savage VR, Kolb B. 2002. Widespread but regionally specific effects of experimenterversus self-administered morphine on dendritic spines in the nucleus accumbens, hippocampus, and neocortex of adult rats. Synapse 46: 271-279.

Russo SJ, Bolanos CA, Theobald DE, DeCarolis NA, Renthal W, Kumar A, Winstanley CA, Renthal NE, Wiley MD, Self DW, et al. 2007. IRS2-Akt pathway in midbrain dopamine neurons regulates behavioral and cellular responses to opiates. Nat Neurosci 10: 93-99.

Russo SJ, Mazei-Robison MS, Ables JL, Nestler EJ. 2009. Neurotrophic factors and structural plasticity in addiction. Neuropharmacology 56 (Suppl 1): 73-82.

Russo SJ, Dietz DM, Dumitriu D, Morrison JH, Malenka RC, Nestler EJ. 2010. The addicted synapse: Mechanisms of synaptic and structural plasticity in nucleus accumbens. Trends Neurosci 33: 267-276.

Saal D, Dong Y, Bonci A, Malenka RC. 2003. Drugs of abuse and stress trigger a common synaptic adaptation in dopamine neurons. Neuron 37: 577-582.

Sarti F, Borgland SL, Kharazia VN, Bonci A. 2007. Acute cocaine exposure alters spine density and long-term potentiation in the ventral tegmental area. Eur J Neurosci 26: 749-756. 
M.S. Mazei-Robison and E.J. Nestler

Sesack SR, Grace AA. 2010. Cortico-Basal Ganglia reward network: Microcircuitry. Neuropsychopharmacology 35: $27-47$.

Shaw-Lutchman TZ, Barrot M, Wallace T, Gilden L, Zachariou V, Impey S, Duman RS, Storm D, Nestler EJ. 2002. Regional and cellular mapping of cAMP response element-mediated transcription during naltrexone-precipitated morphine withdrawal. J Neurosci 22: 3663-3672.

Sklair-Tavron L, Shi WX, Lane SB, Harris HW, Bunney BS Nestler EJ. 1996. Chronic morphine induces visible changes in the morphology of mesolimbic dopamine neurons. Proc Natl Acad Sci 93: 11202-11207.

Spiga S, Serra GP, Puddu MC, Foddai M, Diana M. 2003. Morphine withdrawal-induced abnormalities in the VTA: Confocal laser scanning microscopy. Eur J Neurosci 17: $605-612$.

Swanson LW. 1982. The projections of the ventral tegmental area and adjacent regions: A combined fluorescent retrograde tracer and immunofluorescence study in the rat. Brain Res Bull 9: 321-353.

Torrecilla M, Marker CL, Cintora SC, Stoffel M, Williams JT, Wickman K. 2002. G-protein-gated potassium channels containing Kir3.2 and Kir3.3 subunits mediate the acute inhibitory effects of opioids on locus ceruleus neurons. $J$ Neurosci 22: 4328-4334.

Torrecilla M, Quillinan N, Williams JT, Wickman K. 2008. Pre- and postsynaptic regulation of locus coeruleus neu- rons after chronic morphine treatment: A study of GIRKknockout mice. Eur J Neurosci 28: 618-624.

Van Bockstaele EJ, Reyes BA, Valentino RJ. 2010. The locus coeruleus: A key nucleus where stress and opioids intersect to mediate vulnerability to opiate abuse. Brain Res 1314: $162-174$.

Williams JT, Egan TM, North RA. 1982. Enkephalin opens potassium channels on mammalian central neurones. Nature 299: 74-77.

Williams JT, Bobker DH, Harris GC. 1991. Synaptic potentials in locus coeruleus neurons in brain slices. Prog Brain Res 88: 167-172.

Williams JT, Christie MJ, Manzoni O. 2001. Cellular and synaptic adaptations mediating opioid dependence. Physiol Rev 81: 299-343.

Wolf DH, Numan S, Nestler EJ, Russell DS. 1999. Regulation of phospholipase Cgamma in the mesolimbic dopamine system by chronic morphine administration. J Neurochem 73: $1520-1528$.

Wolf DH, Nestler EJ, Russell DS. 2007. Regulation of neuronal PLCgamma by chronic morphine. Brain Res 1156: 9-20.

Zachariou V, Liu R, LaPlant Q, Xiao G, Renthal W, Chan GC, Storm DR, Aghajanian G, Nestler EJ. 2008. Distinct roles of adenylyl cyclases 1 and 8 in opiate dependence: Behavioral, electrophysiological, and molecular studies. Biol Psychiatry 63: 1013-1021. 


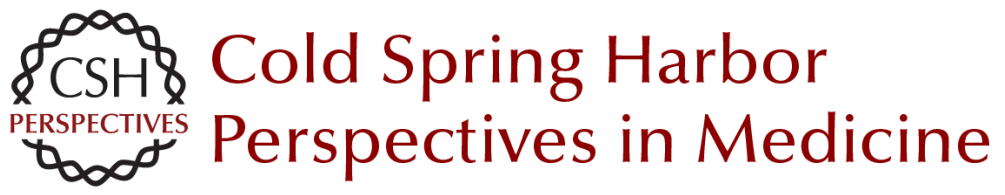

\title{
Opiate-Induced Molecular and Cellular Plasticity of Ventral Tegmental Area and Locus Coeruleus Catecholamine Neurons
}

\author{
Michelle S. Mazei-Robison and Eric J. Nestler
}

Cold Spring Harb Perspect Med 2012; doi: 10.1101/cshperspect.a012070

\section{Subject Collection Addiction}

Developments from Bulk Optogenetics to

Single-Cell Strategies to Dissect the Neural

Circuits that Underlie Aberrant Motivational States

Jose Rodriguez-Romaguera, Vijay M.K.

Namboodiri, Marcus L. Basiri, et al.

Consequences of Parental Opioid Exposure on

Neurophysiology, Behavior, and Health in the

Next Generations

Fair M. Vassoler and Mathieu E. Wimmer

Animal Models of the Behavioral Symptoms of

Substance Use Disorders Louk J.M.J. Vanderschuren and Serge H. Ahmed

Translational Research in Nicotine Addiction Miranda L. Fisher, James R. Pauly, Brett Froeliger, et al.

Neonatal Opioid Withdrawal Syndrome (NOWS): A Transgenerational Echo of the Opioid Crisis Andrew E. Weller, Richard C. Crist, Benjamin C. Reiner, et al.

Impairment of Synaptic Plasticity by Cannabis, $\Delta^{\mathbf{9}}$ -THC, and Synthetic Cannabinoids

Alexander F. Hoffman, Eun-Kyung Hwang and Carl R. Lupica

Drug-Evoked Synaptic Plasticity of Excitatory

Transmission in the Ventral Tegmental Area Camilla Bellone, Michael Loureiro and Christian Lüscher

Opioid-Induced Molecular and Cellular Plasticity

of Ventral Tegmental Area Dopamine Neurons

Marie A. Doyle and Michelle S. Mazei-Robison
The Persistent Challenge of Developing Addiction

Pharmacotherapies

Sarah E. Swinford-Jackson, Charles P. O'Brien,

Paul J. Kenny, et al.

Opioid Modulation of the Gut-Brain Axis in Opioid-Associated Comorbidities

Li Zhang and Sabita Roy

Epigenetics of Drug Addiction

Andrew F. Stewart, Sasha L. Fulton and lan Maze

Genetic Vulnerability to Opioid Addiction

Brian Reed and Mary Jeanne Kreek

Glutamatergic Systems and Memory Mechanisms Underlying Opioid Addiction

Jasper A. Heinsbroek, Taco J. De Vries and Jamie Peters

Mechanisms of Nicotine Addiction

Marina R. Picciotto and Paul J. Kenny

Neural Substrates and Circuits of Drug Addiction Matthew W. Feltenstein, Ronald E. See and Rita A. Fuchs

The Role of the Central Amygdala in Alcohol

Dependence Marisa Roberto, Dean Kirson and Sophia Khom

For additional articles in this collection, see http://perspectivesinmedicine.cshlp.org/cgi/collection/ 